\title{
Etude des effets parasites affectant le fonctionnement des transistors à effet de champ à gaz d'électrons bidimensionnel
}

\author{
S. Mottet $\left({ }^{1}\right)$, J. M. Dumas $\left({ }^{1}\right)$, J. E Viallet $\left({ }^{1}\right)$, A. Belhadj $\left({ }^{1}\right)$ et P. Audren $\left({ }^{2}\right)$ \\ ( $\left.{ }^{1}\right)$ Centre National d'études des Télécommunications, 22301 Lannion Cedex, France \\ (2) Institut Universitaire de Technologie, 22302 Lannion Cedex, France
}

(Reçu le 18 novembre 1988, accepté le 6 mars 1989)

\begin{abstract}
Résumé. - Nous avons mené une étude des effets parasites présentés par les transistors à effet de champ à gaz d'électrons bidimensionnel (HEMT). Ces effets sont étroitement liés à la technologie d'élaboration des composants. L'étude expérimentale, couplée à la simulation numérique du dispositif à permis de montrer l'origine physique de ces effets : le dopage résiduel de la couche de GaAs y joue un rôle prépondérant. L'étude montre également la sensibilité de ces effets aux paramètres technologiques de croissance des couches.
\end{abstract}

\begin{abstract}
A study of the parasitic effects detrinental to the operation of two-dimensional electron gas field effect transistors (HEMTs) has been carried-out. These effects are strongly process-dependent. An experimental investigation and numerical simulations of the device permit the physical origin of these effects to be demonstrated : the residual doping level of the GaAs layer is a main parameter. We equally show that the epilayer growth parameters strongly influence on these effects.
\end{abstract}

\section{Introduction.}

Nous avons effectué une étude des effets parasites présentés par les transistors à effet de champ à gaz d'électrons bidimensionnel (HEMT). Ces effets sont étroitement liés à la technologie d'élaboration des composants mais peuvent également dépendre de la structure même du dispositif. Ces effets peuvent pénaliser les performances microondes des dispositifs discrets ou en limiter les possibilités d'intégration [1]. Notre étude porte sur des composants issus de différents processus de fabrication et de différents constructeurs. Les transistors que nous avons caractérisés ne présentent pas de commutation retardée de grille, effet parasite souvent observé par le passé sur les MESFETs. Ceci signifie que les améliorations technologiques menées sur les transistors à effet de champ à grille Schottky sur GaAs (MESFET) ont été transférées avec succés au HEMT, pour ce qui relève de la préparation et la passivation de la surface. Toutefois les effets parasites pouvant être attribués au volume, tels les effets de coude (kink effect) et de commmande par le substrat (backgating), sont encore observés sur les caractéristiques des HEMTs. D'autres effets, en particulier ceux inhérents à la présence des centres DX dans AlGaAs, peuvent être observés aux basses températures : effondrement du réseau de caractéristiques, décalage de la tension de seuil, photoconductivité persistante... Ces derniers effets sont sensibles à la topologie du composant dans l'espace drain source et sont actuellement l'objet d'études.

Dans la présente contribution nous présentons des résultats expérimentaux représentatifs du comportement des dispositifs présentant les effets de coude et de commande par le substrat : effets liés au substrat. Un travail de modélisation et simulation numérique a ensuite servi de base à l'interprétation de l'origine physique de ces effets, ainsi que nous l'avions fait pour les MESFETs [2].

\section{Résultats expérimentaux.}

1.1 STRUCTURE DES DisPositifs ÉTUdiés. - La figure 1 représente le schéma de la structure des dispositifs étudiés, réalisés en épitaxie par jets moléculaires. Sur un substrat GaAs semi-isolant non intentionnellement dopé est épitaxiée une couche de GaAs non dopé que l'on sait être résiduelle de type $p$ pour ce type d'épitaxie [3]. Sur cette couche de 


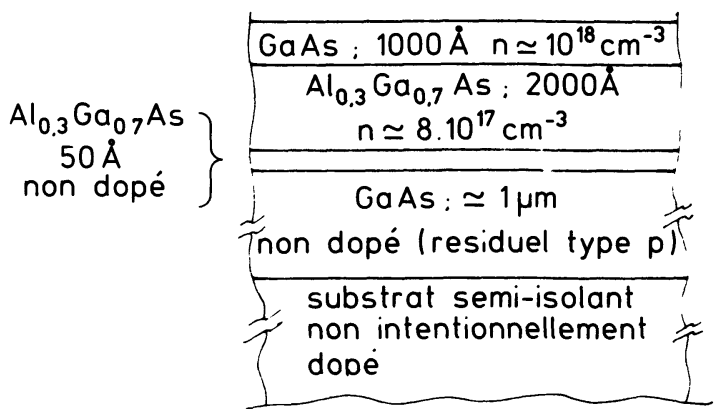

Fig. 1. - Structure du transistor développée en épitaxie par jets moléculaires.

[HEMT structure grown by molecular beam epitaxy.]

GaAs est épitaxiée une couche de $\mathrm{Al}_{0,3} \mathrm{Ga}_{0,7} \mathrm{As}$, de $50 \AA$ d'épaisseur, constituant le «spacer ». La couche qui permet la constitution du gaz d'électrons est du $\mathrm{Al}_{0,3} \mathrm{Ga}_{0,7} \mathrm{As}$ de type $\mathrm{n}$ dopé Si à $8 \times 10^{17} \mathrm{~cm}^{-3}$ (donnée du constructeur) et de $2000 \AA$ d'épaisseur. La grille de type creusée (recess) est déposée sur cette couche. Elle est constituée de $\mathrm{TiAl}$, a une longueur de $0,5 \mu \mathrm{m}$ et une largeur de $300 \mu \mathrm{m}$. Les contacts ohmiques de source et de drain sont en AuGeNi surépaissis TiPtAu. Ces contacts ohmiques sont alliés sur une couche de GaAs de type $\mathrm{n}^{+}$épitaxiée sur AlGaAs. Ce transistor présente un Facteur de Bruit typique de 1,3 dB avec un Gain Associé de $10,5 \mathrm{~dB}$ à $12 \mathrm{GHz}$.

\subsection{MISE EN ÉVIDENCE EXPÉRIMENTALE DES EFFETS} PARASITES. - La figure $2 b$ représente le réseau de caractéristiques courant-drain $I_{\mathrm{DS}}$ en fonction de la tension drain-source $V_{\text {Ds }}$ pour différentes valeurs de la tension grille-source $V_{\mathrm{GS}}$. L'effet de coude correspond à l'augmentation de courant-drain dans la zone de saturation, à partir de la «tension de coude » $V_{\mathrm{Ds}}^{\mathrm{k}}$. Dans le cas présent $V_{\mathrm{DS}}^{\mathrm{k}} \mathrm{s}^{\prime}$ étend de $\simeq 3 \mathrm{~V}$ à $\simeq 3,4 \mathrm{~V}$ selon la valeur de $V_{\mathrm{GS}}$. Cet effet parasite a d'abord été observé sur les MOSFETs silicium [4] et est encore aujourd'hui sujet d'études [5]. Il affecte également les MESFETs GaAs [1,6]. Le pic observé sur la conductance de sortie $g_{\mathrm{d}}$ en fonction de $V_{\mathrm{DS}}$ et $V_{\mathrm{GS}}$ (Fig. 2a) permet de mieux visualiser l'effet de coude. Dans les MESFETs nous avions montré que l'effet de coude était lié au comportement du substrat semi-isolant ce qui pouvait être confirmé expérimentalement par la mesure du courant de substrat [6]. La figure $2 c$ représente le courant-substrat $I_{\text {SUB }}$ en fonction de $V_{\text {DS }}$ et de $V_{\mathrm{GS}}$. Elle montre une augmentation du courant lorsque $V_{\text {DS }}$ dépasse $V_{\text {DS }}^{\mathrm{k}}$. Ce courant, compte-tenu de la structure, est un courant de trous. Ce résultat est très proche de celui observé sur les MESFETs GaAs. Dans le cas des MESFETs, l'interprétation de l'origine de l'excès de courant dans le substrat semi-isolant, repose sur une multiplication des por-

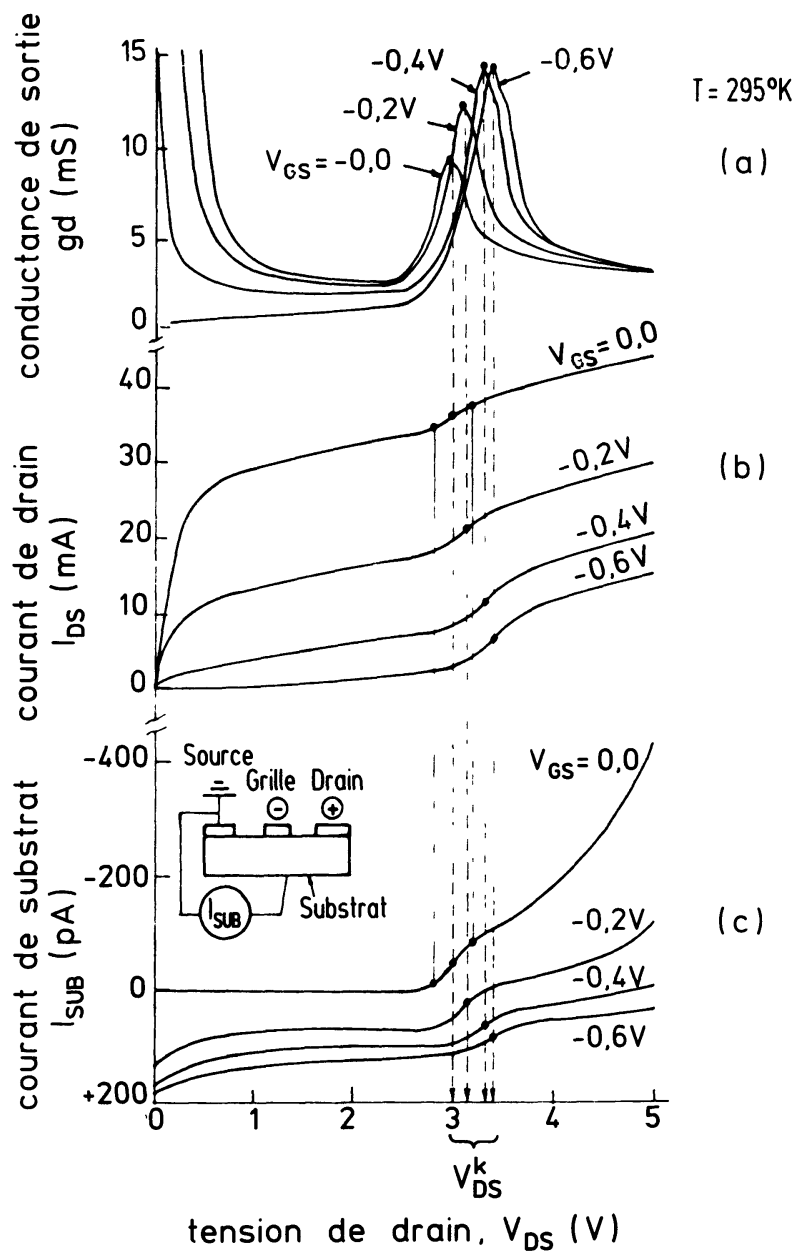

Fig. 2. - a) Conductance de sortie $g_{\mathrm{d}}$ en fonction des tensions drain-source $V_{\mathrm{DS}}$ et grille-source $V_{\mathrm{Gs}}$. Le pic observé sur $\boldsymbol{g}_{\mathrm{d}}$ permet de visualiser facilement la tension de coude $V_{\mathrm{DS}}^{\mathrm{k}}$. b) Caractéristique courant-drain $I_{\mathrm{DS}}$ en fonction des tensions drain-source $V_{\mathrm{DS}}$ et grille-source $V_{\mathrm{GS}}$. c) Courant de substrat $I_{\mathrm{SUB}}$ en fonction des tensions drain-source $V_{\mathrm{DS}}$ et grille-source $V_{\mathrm{GS}}$. Source et substrat sont courtcircuités. L'augmentation observée sur $I_{\text {SUB }}$ correspond parfaitement à celle observée sur $I_{\mathrm{DS}}$, comme indiqué pour $V_{\mathrm{GS}}=0$.

[a) Output conductance $g_{\mathrm{d}}$ versus drain to source voltage $V_{\mathrm{DS}}$ and gate to source voltage $V_{\mathrm{Gs}}$ characteristics. The abrupt increase on $g_{\mathrm{d}}$ easily visualizes the kink voltage $V_{\mathrm{DS}}^{\mathrm{k}}$. b) Drain current $I_{\mathrm{DS}}$ versus drain to source voltage $V_{\mathrm{DS}}$ and gate to source voltage $V_{\mathrm{GS}}$ characteristics. c) Substrate current $I_{\mathrm{SUB}}$ versus drain to source voltage $V_{\mathrm{DS}}$ and gate to source voltage $V_{\mathrm{GS}}$ characteristics. The abrupt increase on $I_{\mathrm{SUB}}$ corresponds to the increase on $I_{\mathrm{DS}}$ as indicated for $V_{\mathrm{GS}}=0$.]

teurs dans le canal résultant d'un mécanisme d'ionisation par impact [6,7]. Dans le cas présent, le coefficient en température, positif, de $V_{\mathrm{DS}}^{\mathrm{k}}$ (Fig. 3) indique que ce mécanisme est de nouveau présent $[8,9]$. Dans le canal, qu'il s'agisse d'un MESFET ou d'un HEMT, les porteurs majoritaires sont des électrons. Une multiplication très faible ne joue pas 


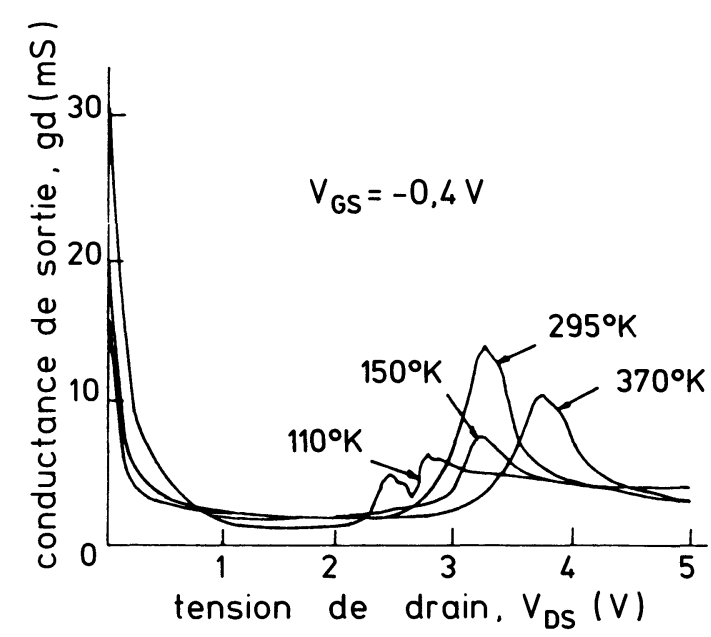

Fig. 3. - Conductance de sortie $g_{\mathrm{d}}$ en fonction de $V_{\mathrm{DS}}$ et de la température, pour $V_{\mathrm{GS}}=-0,4 \mathrm{~V}$.

[Output conductance $g_{\mathrm{d}}$ versus $V_{\mathrm{DS}}$, as a function of temperature, for $V_{\mathrm{GS}}=-0.4 \mathrm{~V}$.]

sur la densité d'électrons et, par conséquent, n'est guère décelable sur le courant-drain. Par contre à tout électron généré correspond un trou, ce qui peut augmenter sensiblement la population de porteurs minoritaires. Les trous ne sont pas confinés dans le canal. Au contraire tout excès de trous est éjecté du canal soit vers la zone de charge d'espace de grille ou vers le substrat.

Cet excès de porteurs minoritaires dans le canal ne suffit pas à expliquer à lui seul les variations de conductance mesurées. Le courant-substrat est extrèmement faible comparé au courant-drain : l'augmentation de conductance n'est pas liée à la seule multiplication des porteurs majoritaires.

En fait on peut vérifier qu'il n'est pas nécessaire d'avoir un excès de porteurs pour moduler la conductance, en mesurant l'influence de la polarisation du substrat sur le courant-drain. La figure 4 représente les mêmes caractéristiques que celles représentées sur la figure $2 \mathrm{~b}$ mais pour deux polarisations du substrat différentes : $V_{\text {SUB }}=0$ (identique à la figure $2 b)$ et $V_{\text {SUB }}=-15 \mathrm{~V}$ par rapport à la source. Pour $V_{\text {DS }}<V_{\text {DS }}^{\mathrm{k}}$ on observe une diminution du courant $I_{\mathrm{DS}}$ lorsqu'une tension négative est appliquée sur le substrat. De même sur la figure 5 nous observons une diminution de la transconductance totale $g_{\mathrm{m}}$. Ces résultats indiquent une diminution de la densité surfacique du gaz d'électrons bidimensionnel. Pour $V_{\mathrm{DS}}>V_{\mathrm{DS}}^{\mathrm{k}}$ le courant $I_{\mathrm{DS}}$ est indépendant de $V_{\text {SUB }}$.

Ces résultats expérimentaux peuvent être interprétés comme suit :

Pour $V_{\mathrm{SUB}}<0$ et $V_{\mathrm{DS}}<V_{\mathrm{DS}}^{\mathrm{k}}$ la jonction $\mathrm{n}$ AlGaAs/p GaAs est polarisée en inverse et provoque une diminution de la densité de porteurs du gaz d'électrons.

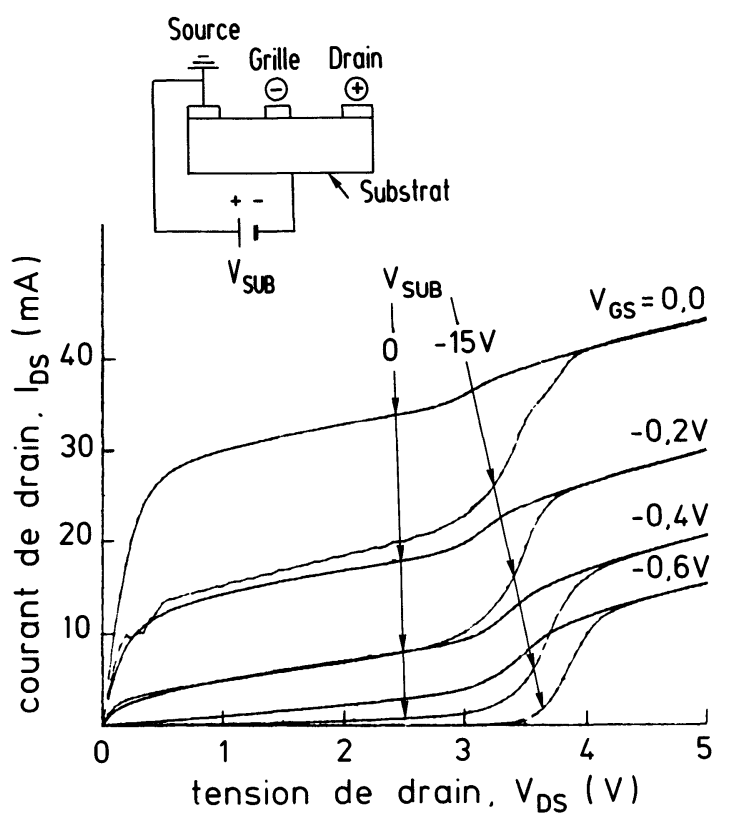

Fig. 4. - Mêmes caractéristiques que figure $2 b$, montrant l'influence sur $I_{\mathrm{DS}}$ d'une tension substrat-source, $V_{\mathrm{SUB}}$, négative.

[Same characteristics as figure $2 b$, showing the influence of negative substrate to source voltage, $V_{\mathrm{SUB}}$, on $I_{\mathrm{DS}}$.]

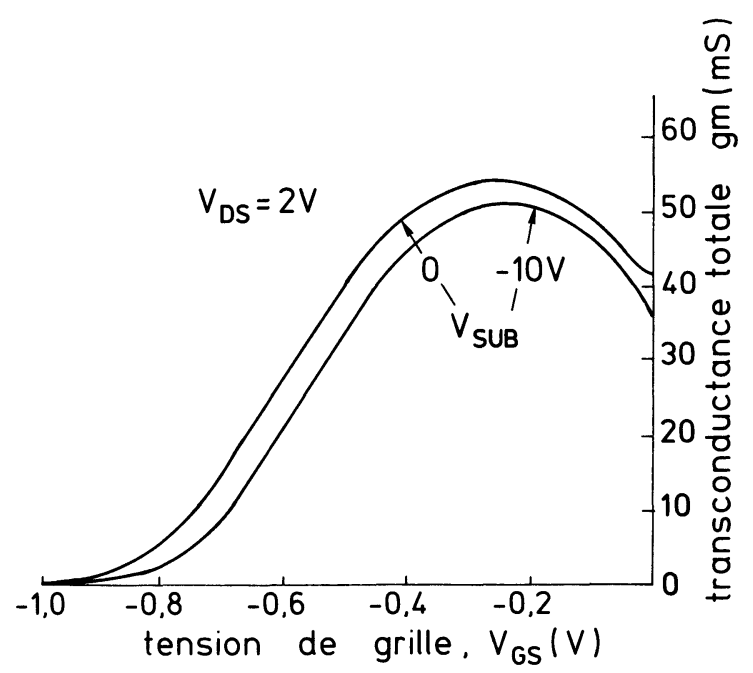

Fig. 5. - Variation de la transconductance totale $g_{\mathrm{m}}$ en fonction des tensions $V_{\mathrm{GS}}$ et $V_{\mathrm{SUB}}$, pour $V_{\mathrm{DS}}<V_{\mathrm{DS}}^{\mathrm{k}}$.

[Total transconductance $g_{\mathrm{m}}$ variation as a function of $V_{\mathrm{GS}}$ and $V_{\mathrm{SUB}}$ voltages, for $V_{\mathrm{DS}}<V_{\mathrm{DS}}^{\mathrm{k}}$.]

Pour $V_{\text {SUB }}<0$ et $V_{\text {DS }}>V_{\text {DS }}^{\mathrm{k}}$ l'excès de porteurs minoritaires générés par ionisation par impact permet au gaz d'électrons de retrouver la densité de porteurs présente en l'absence de polarisation négative de substrat. L'excès de porteurs minoritaires générés est responsable de l'excès de courant de substrat mesuré. On constate d'ailleurs sur la figure 


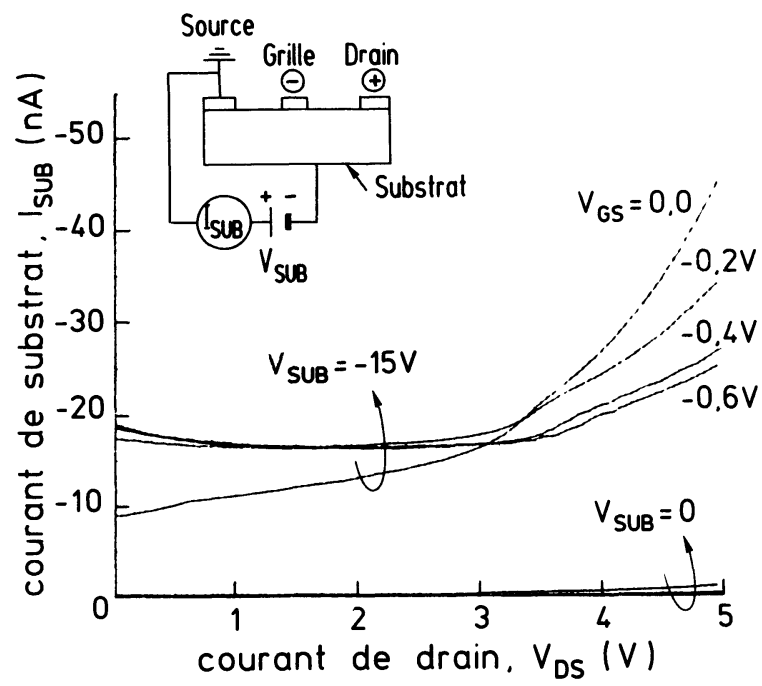

Fig. 6. - Courant substrat $I_{\text {SUB }}$ en fonction des tensions $V_{\mathrm{DS}}$ et $V_{\mathrm{GS}}$, pour $V_{\mathrm{SUB}}=0$ et $-15 \mathrm{~V}$.

[Substrate to source current $I_{\text {SUB }}$ as a function of $V_{\mathrm{DS}}$ and $V_{\mathrm{GS}}$ voltages for $V_{\text {SUB }}=0$ and $-15 \mathrm{~V}$.]

6 que le courant de substrat $I_{\text {SUB }}$ est d'autant plus élevé que $V_{\text {SUB }}$ est négatif.

- Il a été vérifié que $I_{\mathrm{DS}}$ augmente sans pour autant supprimer l'effet de coude pour $V_{\text {SUB }}>0$ dans le domaine $V_{\mathrm{DS}}<V_{\mathrm{DS}}^{\mathrm{k}}$ et est identique au-delà de $V_{\mathrm{DS}}^{\mathrm{k}}$.

Ces résultats sont très similaires à ceux observés $[1,6,7]$ sur le MESFET GaAs.

\section{Analogie de comportement entre MESFET et HEMT.}

Dans le cas du MESFET GaAs nous avions montré que l'effet de coude est lié directement à la zone de charge d'espace à l'interface de la couche active (ou couche tampon) avec le substrat semi-isolant. L'extension de cette zone de charge d'espace dans la couche active est directement dépendante de la différence de potentiel entre le drain et le substrat [6]. En dehors de l'effet de coude, ce phénomène est responsable des effets de commande par le substrat : diminution de $I_{\mathrm{DS}}$ pour une tension de substrat $V_{\text {SUB }}<0$. Cet aspect physique du rôle joué par la zone de charge d'espace existant entre couche active et substrat semi-isolant, est aujourd'hui bien établi dans le MESFET [10]. Dans le cas de l'effet de coude, l'excès de trous générés au-dessus de $V_{\mathrm{DS}}^{\mathrm{k}}$ provoque une chute de potentiel dans le substrat induisant une diminution de l'extension de la zone de charge d'espace dans le canal et donc la récupération du courant de drain qui devient alors indépendant de $V_{\text {SUB }}$. En l'absence de polarisation de substrat un effet de coude est néanmoins observé : Appliquer une polarisation $V_{\text {SUB }}<0$ ne fait qu'amplifier le phénomène. Ceci est dû au fait que la zone de charge d'espace entre couche active et substrat dépend, sous le drain et donc près de la zone de pincement, de la différence de potentiel entre drain et substrat qui est donc égale à $-V_{\text {DS }}$ pour $V_{\text {SUB }}=0$ car $V_{\text {SUB }}$ est référenciée par rapport à la source.

Pour les HEMTs le problème est plus délicat du fait des hétérostructures et de la conduction par gaz d'électrons bidimensionnel. Par analogie avec le MESFET, on aurait tendance à admettre qu'une différence de potentiel appliquée entre le drain et le substrat peut moduler «l'épaisseur » de la couche active, ou plus précisément, dans le cas présent, la densité surfacique du gaz d'électrons. Dans ce cas il existerait une similitude complète entre les deux types de composants en ce qui concerne les effets du substrat et l'effet de coude. C'est cette similitude qui a guidée notre étude sur l'équilibre entre les différentes couches du HEMT en fonction de la différence de potentiel entre drain et substrat.

\section{Sensibilité du gaz d'électrons à la polarisation drain-substrat.}

3.1 Modèle NUMÉRIQUe. - Pour étudier la sensibilité du gaz d'électrons (en terme de densité surfacique) à la polarisation drain-substrat, nous avons utilisé la simulation numérique. Celle-ci est basée sur la résolution des équations décrivant le transport des porteurs dans les hétérostructures.

Nous rappelons ici le système général d'équations utilisé pour décrire les hétérojonctions en régime stationnaire en l'absence de porteurs thermalisés:

$$
\left\{\begin{aligned}
\operatorname{div}(\varepsilon \cdot \operatorname{grad} \varphi) & =q \cdot(n-p-C) \\
-\frac{1}{q} \cdot \operatorname{div} \mathrm{J}_{\mathrm{n}} & =-U \\
\frac{1}{q} \cdot \operatorname{div} \mathrm{J}_{\mathrm{p}} & =-U
\end{aligned}\right.
$$

où $U$ est le terme recombinaison génération et $C$ la densité de charges fixes. Pour la plupart des matériaux dopés par des impuretés peu profondes, $C$ ne dépend que de la densité d'impuretés. Dans le cas de matériaux semi-isolants $C$ est également fonction des densités locales de porteurs libres. A partir de l'équation de transport de Boltzmann, il peut être établi que le courant d'entraînement-diffusion dans des hétérojonctions isothermes peut s'exprimer sous la forme suivante [11], indépendamment de la statistique utilisée pour décrire les densités de porteurs $\mathrm{n}$ et $\mathrm{p}$ :

$$
\left\{\begin{array}{l}
\mathbf{J}_{\mathrm{n}}=n \cdot \mu_{\mathrm{n}} \cdot \operatorname{grad} E_{\mathrm{Fn}} \\
\mathbf{J}_{\mathrm{p}}=p \cdot \mu_{\mathrm{p}} \cdot \operatorname{grad} E_{\mathrm{Fp}}
\end{array}\right.
$$

où $E_{\mathrm{Fn}}$ et $E_{\mathrm{Fp}}$ sont les potentiels électrochimiques des électrons et des trous, $\mu_{\mathrm{n}}$ et $\mu_{\mathrm{p}}$ leurs mobilités respectives. 
Du fait que dans les HEMTs il existe une accumulation de porteurs (gaz d'électrons) à l'interface $\mathrm{AlGaAs} / \mathrm{GaAs}$, les densités de porteurs doivent expressément être décrites par la statistique de Fermi-Dirac. Dans l'hypothèse des bandes paraboliques, il vient :

$$
\left\{\begin{array}{l}
n=N_{\mathrm{c}} \cdot \mathcal{F}_{1 / 2}\left[-\frac{E_{\mathrm{c}}-E_{\mathrm{Fn}}}{k \cdot T}\right] \\
p=N_{\mathrm{v}} \cdot \mathcal{F}_{1 / 2}\left[\frac{E_{\mathrm{v}}-E_{\mathrm{Fp}}}{k \cdot T}\right]
\end{array}\right.
$$

où $\mathcal{F}_{1 / 2}$ est l'intégrale de Fermi d'ordre $1 / 2, N_{\mathrm{c}}$ et $N_{\mathrm{v}}$ les densités d'état effectives dans les bande de conduction et de valence repérées par les énergies de leurs extréma $E_{\mathrm{c}}$ et $E_{\mathrm{v}}$. Le terme classique de recombinaison génération de Shockley-Hall-Read, $U$, est habituellement exprimé en statistique de Maxwell-Boltzmann. Il peut être généralisé à la statistique de Fermi-Dirac. Pour un centre de recombinaison possèdant un niveau d'énergie $E_{\mathrm{T}}$ dans la bande interdite, il vient [11]:

$$
\begin{gathered}
U=\frac{n \cdot p-n_{1} \cdot p_{1}}{\tau_{\mathrm{n}} \cdot\left(p+p_{1}\right)+\tau_{\mathrm{p}} \cdot\left(n+n_{1}\right)} \\
\left\{\begin{array}{l}
n_{1}=n \cdot \exp \left[\frac{E_{\mathrm{T}}-E_{\mathrm{Fn}}}{k \cdot T}\right] \\
p_{1}=p \cdot \exp \left[\frac{E_{\mathrm{Fp}}-E_{\mathrm{T}}}{k \cdot T}\right]
\end{array}\right.
\end{gathered}
$$

L'expression générique des densités de charge fixes est : $C=\sum\left(N_{\mathrm{D}}^{+}-N_{\mathrm{A}}^{-}\right)$. Dans le matériau semiisolant l'ionisation du centre profond de compensation dépend des densités de porteurs libres [12]. Dans le cas présent le substrat est du GaAs semiisolant non dopé pour lequel la compensation des niveaux résiduels accepteurs peu profonds est obtenue par le niveau donneur profond $E_{\mathrm{L} 2}$ situé près du milieu de la bande interdite. Dans ce cas $N_{\mathrm{A}}^{-} \equiv N_{\mathrm{A}}$ et

$$
\begin{aligned}
N_{\mathrm{D}}^{+}= & N_{\mathrm{D}} \cdot \frac{C_{\mathrm{p}} \cdot p-C_{\mathrm{n}} \cdot n_{1}}{C_{\mathrm{p}} \cdot\left(p+p_{1}\right)+C_{\mathrm{n}} \cdot\left(n+n_{1}\right)} \\
& \left\{\begin{array}{l}
n_{1}=n \cdot \exp \left[\frac{E_{\mathrm{L} 2}-E_{\mathrm{Fn}}}{k \cdot T}\right] \\
p_{1}=p \cdot \exp \left[\frac{E_{\mathrm{Fp}}-E_{\mathrm{L} 2}}{k \cdot T}\right]
\end{array}\right.
\end{aligned}
$$

avec $C_{n}$ et $C_{p}$ les coefficients de capture des électrons et des trous. Cette formulation est totalement compatible avec l'utilisation de la statistique de Fermi-Dirac.

La résolution numérique de ce système d'équations est obtenue par une méthode de différences finies [11].

3.2 STRUCTURE SIMULÉE ET CONSTANTES PHYSIQUES. - La structure unidimensionnelle simulée (Tab. I) est représentative de la coupe d'un HEMT

Tableau I. - Valeurs des paramètres dans les différentes couches constituant la structure de base étudiée en simulation numérique.

[Parameter values for the different layers of the structure used for the numerical simulation.]

\begin{tabular}{|c|c|c|c|}
\hline Matériau & Epaisseur & Niveau de dopage & Niveau de vie des porteurs \\
\hline $\mathrm{Al}_{0,3} \mathrm{Ga}_{0,7} \mathrm{As}$ & $0.2 \mu \mathrm{m}$ & $N_{\mathrm{D}}^{+}=8 \times 10^{17} \mathrm{~cm}^{-3}$ & $\tau_{\mathrm{n}}=\tau_{\mathrm{p}}=10^{-10} \mathrm{~s}$ \\
\hline $\begin{array}{c}\mathrm{Al}_{0,3} \mathrm{Ga}_{0,7} \mathrm{As} \\
\text { spacer }\end{array}$ & $50 \AA$ & $N_{\mathrm{D}}^{+}=10^{14} \mathrm{~cm}^{-3}$ & $\tau_{\mathrm{n}}=\tau_{\mathrm{p}}=10^{-9} \mathrm{~s}$ \\
\hline $\begin{array}{c}\text { GaAs } \\
\text { non dopé }\end{array}$ & $1 \mu \mathrm{m}$ & $N_{\mathrm{A}}^{-}=10^{15} \mathrm{~cm}^{-3}$ & $\tau_{\mathrm{n}}=\tau_{\mathrm{p}}=10^{-8} \mathrm{~s}$ \\
\hline $\begin{array}{c}\text { GaAs } \\
\text { semi-isolant } \\
\text { non dopé }\end{array}$ & $10 \mu \mathrm{m}$ & $N_{\mathrm{A}}^{-}=5 \times 10^{15} \mathrm{~cm}^{-3}$ & $\tau_{\mathrm{n}}=\tau_{\mathrm{p}}=10^{-10} \mathrm{~s}$ \\
\hline
\end{tabular}

sous le drain. Nous avons supposé un contact ohmique idéal directement sur la couche de $\mathrm{Al}_{x} \mathrm{Ga}_{1-x} \mathrm{As}$, et négligée la couche de $\mathrm{n}^{+} \mathrm{GaAs}$ qui ne joue aucun rôle dans ce qui suit. Les paramètres physiques des différents matériaux à température ambiante sont ceux couramment admis tant en ce qui concerne le calcul des densités d'état que les valeurs de mobilité. La discontinuité de la bande de conduction entre $\mathrm{Al}_{x} \mathrm{Ga}_{1-x} \mathrm{As}$ et $\mathrm{GaAs}$ est un paramètre critique. La valeur utilisée est : $\Delta E_{\mathrm{c}}=0,65 \Delta E_{\mathrm{g}}$ avec $\Delta E_{\mathrm{g}}=1,34 x[13]$. Concernant le matériau semi-isolant, la valeur du niveau d'éner- 
gie dans la bande interdite et le rapport des coefficients de capture ont été déduits d'une étude antérieure sur les MESFETs GaAs en fonction de la température corrélée à la simulation numérique [14] :

$$
E_{\mathrm{c}}-E_{\mathrm{L} 2}=0,72 \mathrm{eV} \text { avec } C_{\mathrm{n}} / C_{\mathrm{p}}=10 .
$$

La résolution par méthode des différences finies nécessite un maillage adapté pour décrire de façon précise les variations spatiales au voisinage des hétérojonctions. Le raffinement de maillage utilisé pour l'hétérojonction et le spacer est de $2 \AA$.

3.3 RÉSultats de Simulation. - Les valeurs indiquées dans le tableau 1 sont représentatives de la structure standard étudiée. Dans un premier temps le comportement électrique de cette structure a été étudié en fonction de la polarisation. La figure 7 montre le schéma de bande de la structure à l'équilibre thermodynamique et la figure 8 les profils des densités de porteurs $\mathrm{n}$ et $\mathrm{p}$ et de charges fixes $C$ correspondantes. Sur ces figures est mentionnée la densité surfacique calculée d'électrons dans le GaAs à l'hétérojonction : $n_{\mathrm{s}}=9,2 \times 10^{11} \mathrm{~cm}^{-2}$. La figure 9 est un agrandissement de la zone de l'hétérojonction montrant plus clairement la couche de $50 \AA$ du spacer et les profils de densités de porteurs dans cette région. Ces figures mettent en évidence l'équilibre entre les différentes zones de charge d'espace à l'hétérointerface et l'extension dans le substrat semi-isolant. Pour le substrat semiisolant nous rappelons que le calcul de la densité de charges fixes est dépendant de l'équilibre entre le centre $E_{\mathrm{L} 2}$ et les densités de porteurs libres. D'après la figure 8 on peut voir que le semi-isolant présente un équilibre de matériau massif au-delà de $2,5 \mu \mathrm{m}$.

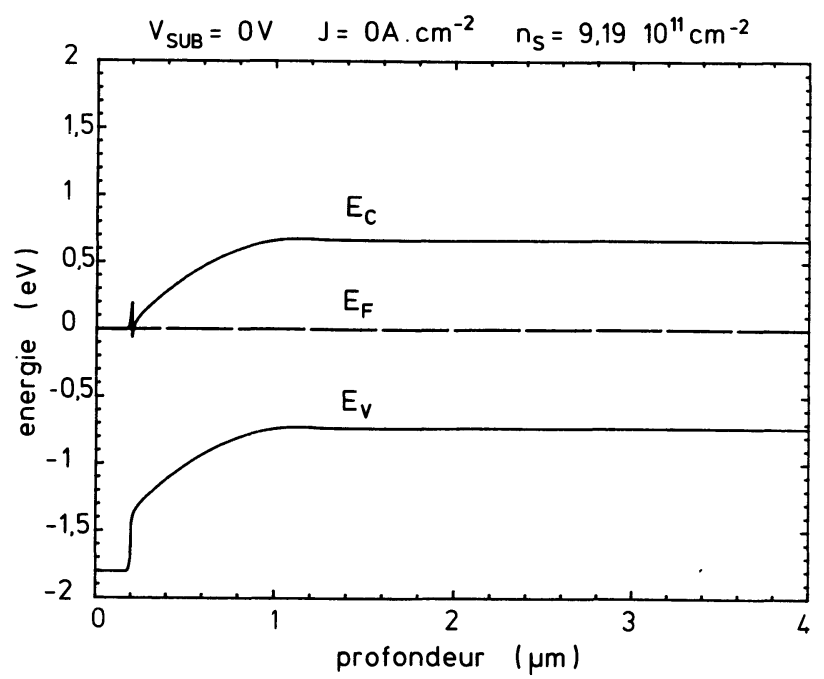

Fig. 7. - Schéma de bande de la structure à l'équilibre thermodynamique.

[Structure band diagram at thermodynamic equilibrium.]

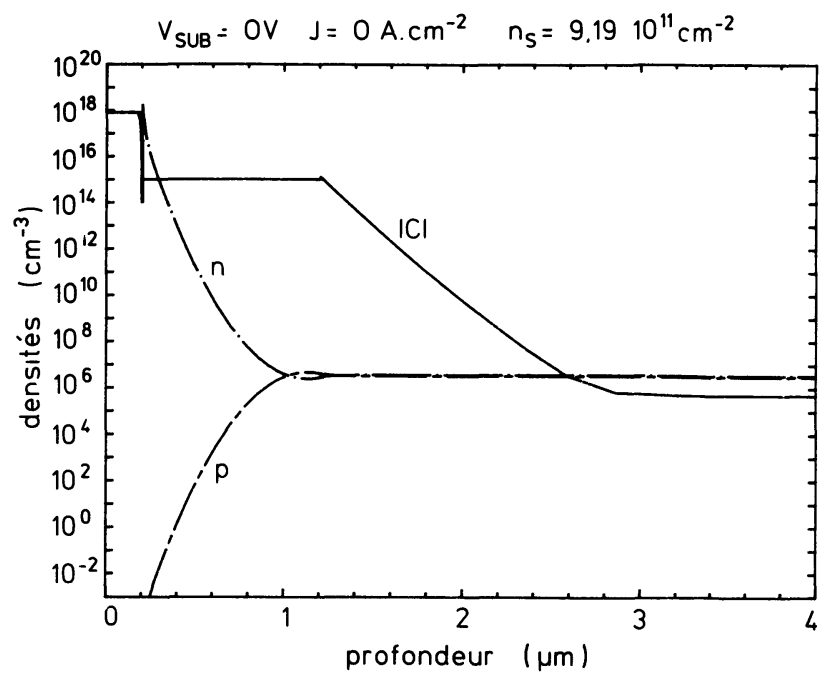

Fig. 8. - Profils des densités de porteurs libres et de charges fixes à l'équilibre thermodynamique. Les traits mixtes correspondent aux densités d'électrons et de trous. La valeur absolue de la densité de charges fixes $|C|$ est tracée en trait continu. La variation de $|C|$ dans le semiisolant, à l'interface avec le GaAs non dopé, provient de la variation de l'ionisation du centre profond $E_{\mathrm{L} 2}$ et correspond à l'extension de la zone de charge d'espace dans le matériau semi-isolant.

[Carrier and fixed charge density profiles at thermodynamic equilibrium. The dashed lines correspond to electron and hole densities. The absolute value of the fixed charge density, $|C|$, is plotted using continuous line. The variation of $|C|$ in the SI at the interface with the undoped $\mathrm{GaAs}$ comes from the variation of the $E_{\mathrm{L} 2}$ deep center ionization and corresponds to the space charge region extend in the SI material.]

La figure 10 représente le schéma de bande de la structure sous une polarisation du substrat de $V_{\text {SUB }}=-2 \mathrm{~V}$. La plus grande partie de la chute de potentiel a lieu dans les $10 \mu \mathrm{m}$ de matériau semiisolant non représentés sur la figure. Cette chute de potentiel correspond au courant de $3,0 \times$ $10^{-6} \mathrm{~A} \cdot \mathrm{cm}^{-2}$ qui circule dans la structure et notamment dans le substrat de haute résistivité. Ce courant prend sa source dans les mécanismes de génération par centres profonds et est directement dépendant de la durée de vie des porteurs dans les différentes couches de la structure comme dans toute jonction polarisée en inverse. Néanmoins une partie de la chute de potentiel se retrouve dans la zone de charge d'espace, matérialisée par la séparation des potentiels électrochimiques des électrons et des trous. L'extension de la zone de charge d'espace sous polarisation du substrat change la conductance du gaz d'électrons en diminuant sa densité jusqu'à $8,9 \times 10^{11} \mathrm{~cm}^{-2}$. La figure 11 montre les profils de densités de porteurs et de charges fixes sous polarisation. Elle montre en particulier les changements d'équilibre au sein du semi-isolant ce qui confirme la 


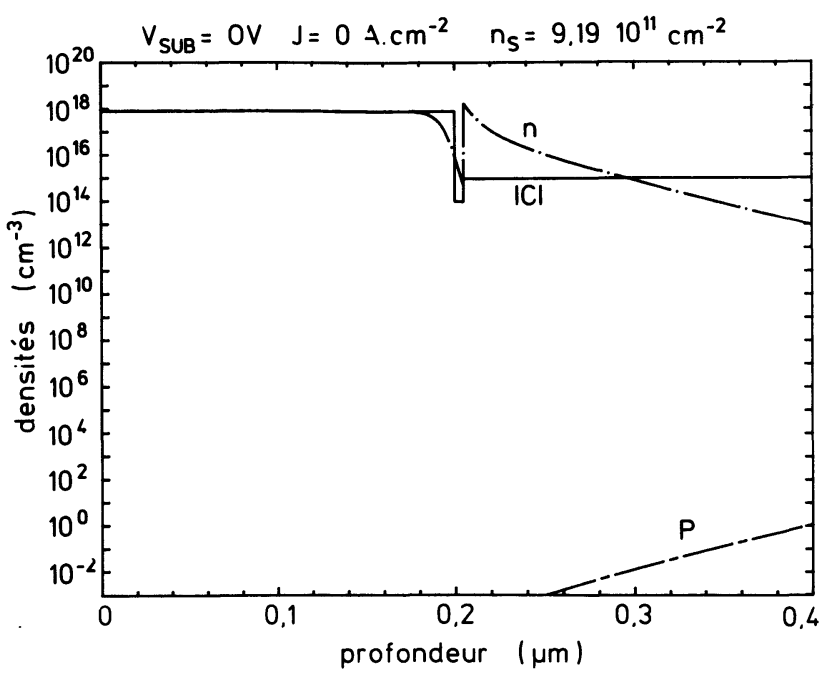

Fig. 9. - Agrandissement des profils de densités de porteurs et de charges fixes à l'hétérojonction, à l'équilibre thermodynamique. La couche "spacer» de AlGaAs correspond à la région dopée à $10^{14} \mathrm{~cm}^{-3}$, à l'interface de l'hétérojonction.

[Magnification of the carrier and fixed charge density profils at the heterostructure at thermodynamic equilibrium. The AlGaAs spacer layer correspond to the $10^{14} \mathrm{~cm}^{-3}$ doping region at the interface of the heterojunction.]

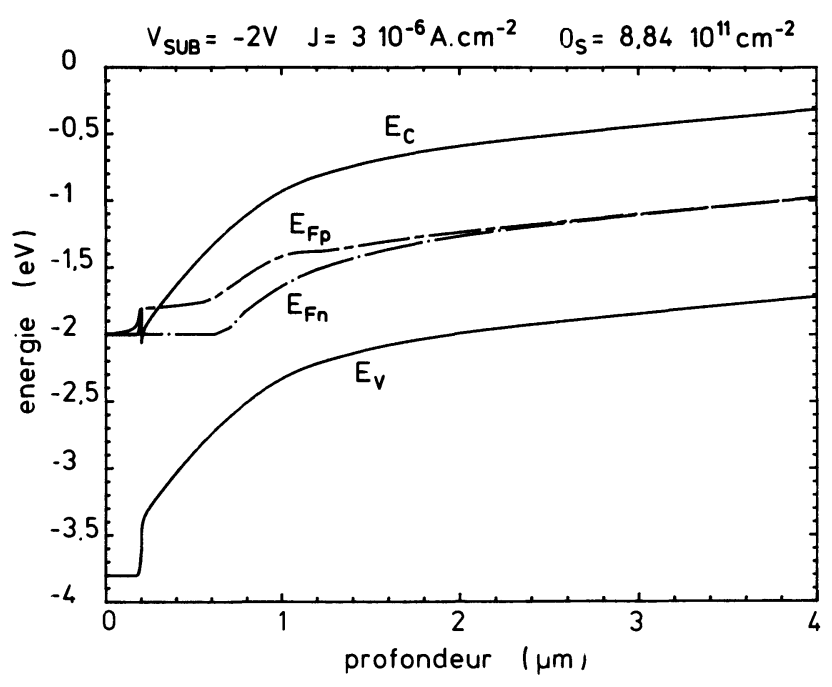

Fig. 10. - Schéma de bande pour $-2 \mathrm{~V}$ de polarisation substrat-drain.

[Band diagram under $-2 \mathrm{~V}$ substrate to drain voltage.]

nécéssité d'une modélisation correcte de ce matériau. Par contre les variations en amplitude du pic d'électrons à l'interface sont difficilement appréciables sans le calcul de la densité surfacique équivalente.

Ces résultats montrent que la densité du gaz d'électrons décroit quand le substrat est polarisé en

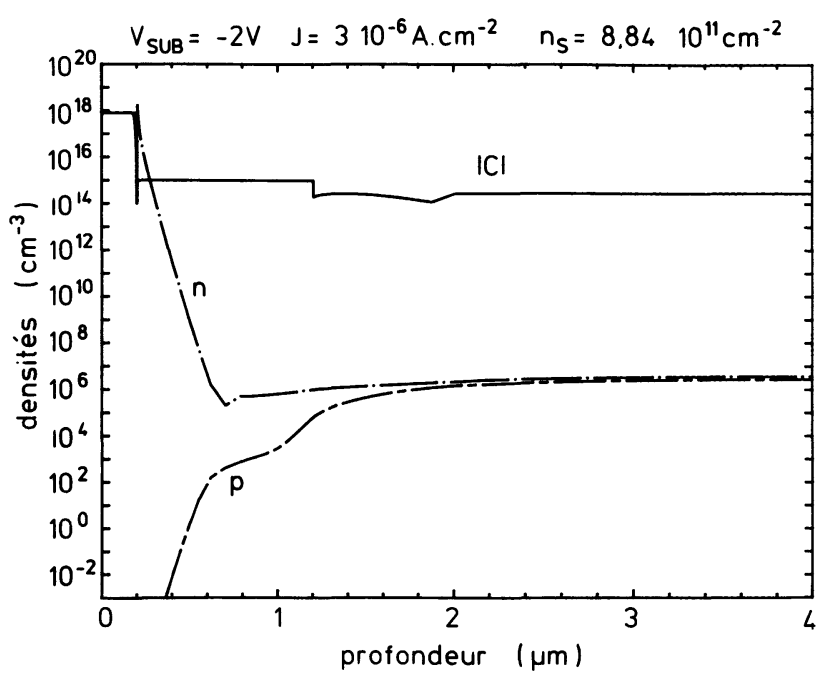

Fig. 11.- Profils de densités de porteurs et de charges fixes pour $-2 \mathrm{~V}$ de polarisation substrat-drain.

[Carrier and fixed charge density profils under $-2 \mathrm{~V}$ substrate to drain voltage.]

inverse. Ceci a lieu sous le drain par auto-polarisation lorsque qu'une polarisation source-drain est appliquée, $V_{\mathrm{DS}}>0$, même si aucune polarisation n'est appliquée effectivement sur le substrat. Il est évident que l'effet est renforcé dès l'instant où une polarisation $V_{\text {SUB }}$ est effectivement appliquée au substrat. Cette étude montre également la chute de potentiel dans le substrat semi-isolant lié au faible courant de diode inverse. Dans ce travail de simulation il n'est pas tenu compte des effets bidimensionnels. Ceux-ci peuvent renforcer l'action d'auto-polarisation. En effet la zone sous le drain d'un HEMT n'est qu'à une longueur de canal de la source et donc à moins d'un micron de la référence de potentiel : la zone sous la source se polarise en directe à concurrence du courant inverse, ce qui apporte une très faible chute de potentiel [6]. La chute de potentiel dans le substrat est dès lors beaucoup plus faible dans le cas de l'auto-polarisation que dans le cas d'une polarisation du substrat qui implique la traversée de l'ensemble du matériau par le courant inverse.

Il est compréhensible que si la génération de porteurs dans le gaz d'électrons augmente, par exemple par l'effet de l'ionisation par impact, alors le courant augmentant, la chute de potentiel finira par avoir totalement lieu dans le substrat laissant la densité du gaz d'électron à sa valeur maximum. Il n'est pas nécessaire de faire appel à une forte polarisation du drain $V_{\mathrm{DS}}>V_{\mathrm{DS}}^{\mathrm{k}}$ pour qu' un excès de porteurs soit généré. L'éclairement de la structure provoque le même phénomène par génération de porteurs par absorption photonique. La lumière a pour effet de supprimer tout effet de polarisation du substrat et l'effet de coude. Ceci confirme le comportement lié au substrat. 
3.4 SENSIBILITÉ AUX PARAMÈTRES TECHNOLOGIQUES DE CROISSANCE. - Il a été montré, sur un exemple type, que la simulation numérique rendait compte de la sensibilité des HEMT à la polarisation du substrat. La simulation est maintenant utilisée pour connaître l'influence des différents paramètres technologiques de la structure sur cet effet, de façon à pouvoir interpréter les différences de comportements qui peuvent exister entre les composants. Pour cela nous avons fait varier la valeur du dopage de la couche de GaAs autour de sa valeur nominale correspondant au tableau I. L'excursion en tension de substrat a été effectuée entre 0 et $-10 \mathrm{~V}$. Les résultats que nous avons obtenus sont synthétisés dans la figure 12 où sont reportées les valeurs prises par la densité surfacique de porteurs dans le gaz d'électrons en fonction de la polarisation pour différents cas de dopage de GaAs. Le dopage de la couche de AlGaAs est de $8 \times 10^{17} \mathrm{~cm}^{-3}$, valeur donnée par le constructeur. Des résultats présentés figure 12 il découle que les HEMTs sont d'autant plus sensibles à la polarisation du substrat que le dopage résiduel de la couche de $\mathrm{GaAs}$ est élevé. Il est à remarquer que les amplitudes de variation que nous avons mesurées, de l'ordre de $30 \%$ de chute de $I_{\text {DSS }}$ pour $V_{\text {SUB }}=-15 \mathrm{~V}$ (Fig. 4$)$, sont tout à fait compatibles avec celles calculées pour des dopages résiduels de $\mathrm{GaAs}$ de 2 à $3 \times 10^{15} \mathrm{~cm}^{-3}$. Ces valeurs de dopage résiduel ne sont pas souhaitées, ni les meilleures que sachent faire les technologues, mais

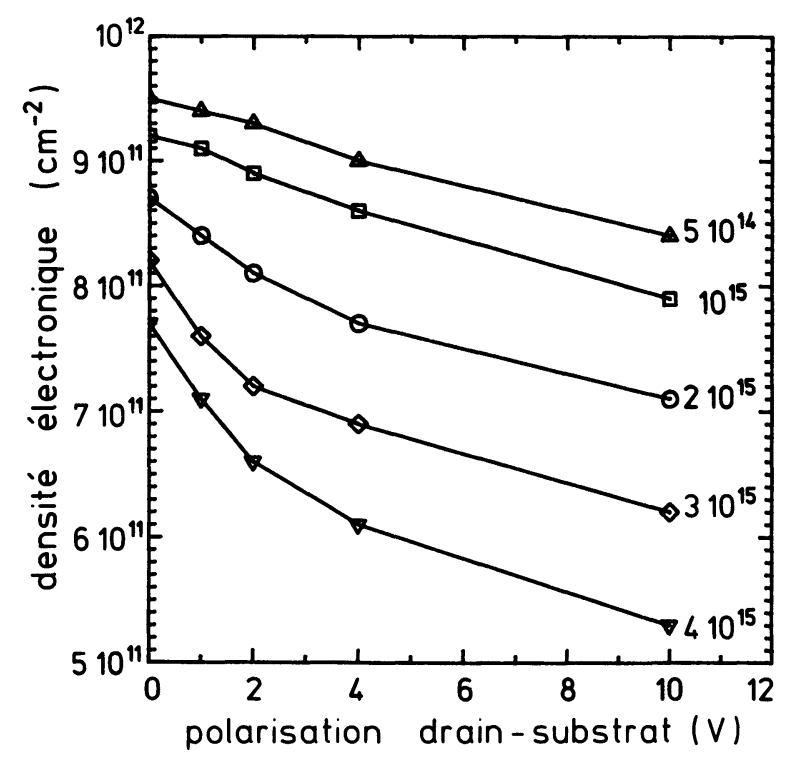

Fig. 12. - Densité surfacique du gaz d'électrons en fonction de l'amplitude de la polarisation substrat-drain pour différentes valeurs du dopage résiduel de la couche de GaAs. Le dopage de AlGaAs est fixé à $8 \times 10^{17} \mathrm{~cm}^{-3}$.

[Electron gas surfacic density versus substrat to drain voltage amplitude, as a function of $\mathrm{GaAs}$ layer residual doping density. The AlGaAs doping density is fixed to $8 \times 10^{17} \mathrm{~cm}^{-3}$.] sont tout à fait réalistes des valeurs effectives pour des couches de l'ordre du micron obtenues industriellement après reprise d'épitaxie. Le dopage résiduel dépend étroitement de la technique d'épitaxie et de sa maîtrise. Pour les épitaxies par jets moléculaires le dopage résiduel est habituellement de type $\mathrm{p}$; la valeur effective de ce dopage près de l'hétéroépitaxie peut être sujette à variation d'une plaquette à l'autre. Il n'est pas évident que les faibles valeurs de dopage résiduel mesurées sur des couches relativement épaisses de GaAs soient éffectivement reproduites dans la couche des HEMT et conservées après reprise d'épitaxie du $\mathrm{AlGaAs}$ : Il est évident qu'à ce niveau de dopage les impuretés résiduelles ou diffusées et les centres complexes issus de leur association avec des défauts cristallins primaires jouent un rôle prédominant. Cette inhomogénéité est illustrée par la figure 13 sur laquelle est tracé le réseau de caractéristiques statiques d'un composant issu de la même plaquette que celui de la figure $2 b$. On constate que ce composant présente un effet de coude très atténué qui correspondrait à un dopage résiduel de GaAs inférieur à $10^{15} \mathrm{~cm}^{-3}$.

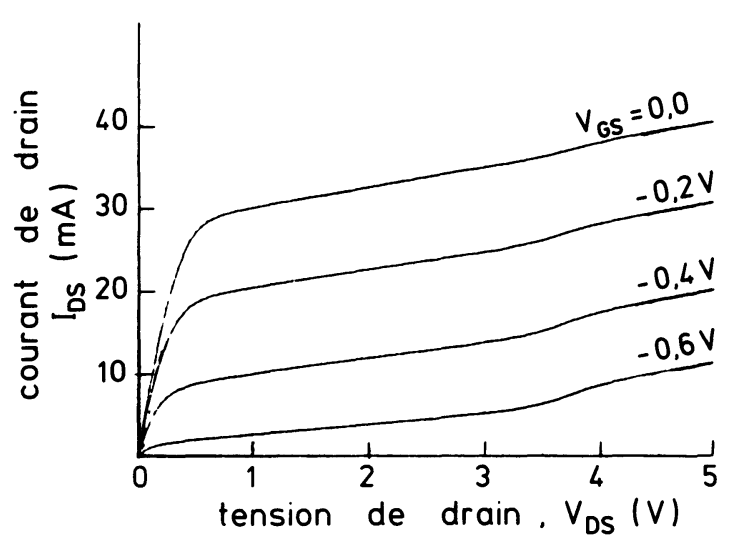

Fig. 13. - Caractéristique courant $I_{\mathrm{Ds}}$ en fonction des tensions $V_{\mathrm{DS}}$ et $V_{\mathrm{GS}}$ pour un transistor issu de la même plaquette que celui représenté figure $2 \mathrm{~b}$.

[Drain current $I_{\mathrm{DS}}$ characteristics versus $V_{\mathrm{DS}}$ and $V_{\mathrm{GS}}$ voltages for a transistor issued from the same batch as the one illustrated figure $2 b$.]

En fait le dopage de la couche de $\mathrm{Al}_{x} \mathrm{Ga}_{1-x} \mathrm{As}$ chez d'autres constructeurs est supérieur à $8 \times 10^{17} \mathrm{~cm}^{-3}$. Etant bien entendu qu'en valeur absolue la densité surfacique électronique est d'autant plus élevée que $\mathrm{Al}_{x} \mathrm{Ga}_{1-x}$ As est dopé, il est néanmoins souhaitable de savoir si ce dopage influe sur l'amplitude des effets de coude. Sur les figures 14 et 15 sont reportées les variations de la densité électronique en fonction de $V_{\text {SUB }}$ pour différentes valeurs du dopage de $\mathrm{AlGaAs}$, pour des dopages résiduels de $10^{15} \mathrm{~cm}^{-3}$ et $3 \times 10^{15} \mathrm{~cm}^{-3}$ respectivement. Ces figures montrent clairement que la densité 


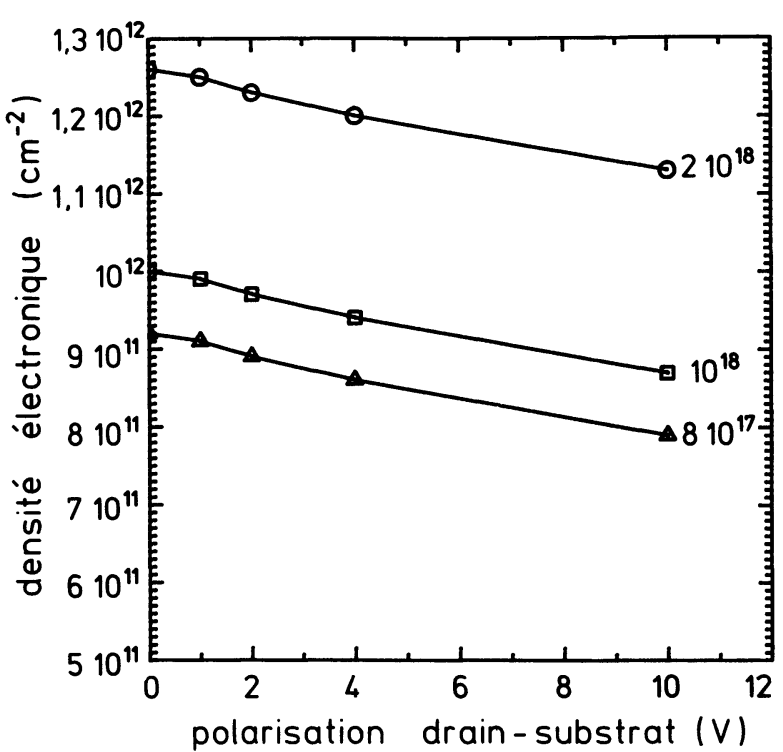

Fig. 14. - Densité surfacique du gaz d'électrons en fonction de l'amplitude de la polarisation substrat-drain pour différentes valeurs du dopage de la couche de AlGaAs. Le dopage résiduel de $\mathrm{GaAs}$ est fixé à $10^{15} \mathrm{~cm}^{-3}$.

[Electron gas surfacic density versus substrat to drain voltage amplitude, as a function of AlGaAs layer doping density. The GaAs residual doping density is fixed to $10^{15} \mathrm{~cm}^{-3}$.]

électronique dépend étroitement du dopage de AlGaAs pour $V_{\text {SUB }}=0$, mais que la variation avec la polarisation ne dépend, elle, que du dopage résiduel comme l'indique le parallèlisme des courbes. Augmenter le dopage de AlGaAs ne diminue donc pas les effets dûs à la polarisation du substrat.

\section{Conclusion.}

La simulation numérique a permis de montrer que les effets parasites que nous avons mis en évidence s'interprètent directement à partir des seules données technologiques de réalisation du transistor. En particulier il ressort des résultats que la sensibilité du courant $I_{\mathrm{DS}}$ à la polarisation du substrat $V_{\text {SUB }}$ et l'effet de coude pour $V_{\mathrm{DS}}>V_{\mathrm{DS}}^{\mathrm{k}}$ sont interprétés dans leur totalité dès l'instant où l'équilibre des différentes couches épitaxiées avec le substrat semiisolant est décrit à partir d'un modèle physique prenant en compte les mécanismes d'ionisation des impuretés et de conduction dans le substrat. Les résultats de la simulation de la sensibilité à l'effet de polarisation du substrat en fonction des dopages des couches a permis de montrer en particulier l'importance du dopage résiduel du GaAs et ses inhomogénéités.

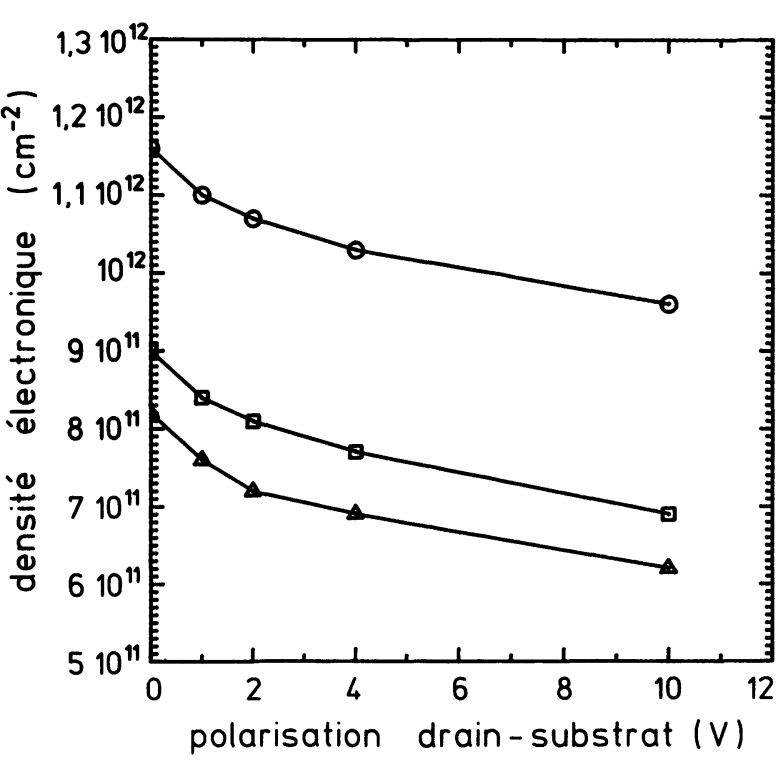

Fig. 15. - Densité surfacique du gaz d'électrons en fonction de l'amplitude de la polarisation substrat-drain pour différentes valeurs du dopage de la couche de AlGaAs. Le dopage résiduel de $\mathrm{GaAs}$ est fixé à $3 \times 10^{15} \mathrm{~cm}^{-3}$.

[Electron gas surfacic density versus substrat to drain voltage amplitude, as a function of $\mathrm{AlGaAs}$ layer doping density. The GaAs residual doping density is fixed to $3 \times 10^{15} \mathrm{~cm}^{-3}$.]

Il est à remarquer de l'ensemble de cette étude qui met en jeu des propriétés de conduction dans les couches épitaxiées et le substrat ne fait pas mention du centre DX auquel est attribué une majorité des effets observés à basse température [15]. Notre propos n'est pas de minorer les effets que peut avoir ce centre à basse température, mais au niveau des effets parasites observés à température ambiante il n'intervient pas. Dans cet ordre d'idée, il a été récemment établi que le centre $\mathrm{DX}$ ne perturbait pas les performances hyperfréquences à température ambiante [16].

En guise de conclusion, il est établi que les effets parasites que nous avons mis en évidence sont intrinsèques à la structure et à son élaboration technologique. Aujourd'hui, des actions correctives (désorption laser des substrats, composants multicouches) sont en cours.

\section{Acknowledgments.}

Ce travail a été partiellement soutenu par la COMMUNAUTÉ ÉCOMOMIQUE EUROPÉENNE au travers le contrat ESPRIT n ${ }^{\circ} 1270$ : «Advanced processing technology for GaAs FETs ». Nous tenons à remercier nos partenaires européens au sein de ce contrat pour nos fructueuses et amicales collaborations. 


\section{Bibliographie}

[1] Rocchi M., (invited) Status of the surface and bulk parasitic effects limiting the performances of GaAs I.C.s, Proc. Eur. Solid State Devices Res. Conf., Eds., J. P. Noblanc and J. Zimmermann , (North-Holland, Amsterdam) 1985, pp. 119-138.

[2] Mottet S. and Viallet J. E., Simulation of III-V devices semi- insulating materials, Proc. of Simulation of Semicond. Devices and Processes, Eds., K. Board and D. R. J. Owen (Pineridge press, Swansea) Vol. 2 (1986) 494-507.

[3] Garcia J. C., Beye A. C., Contour J. P., Neu G., MASSIES J. and BARSKY A., Reduce carbon acceptor incorporation in GaAs grown by molecular beam epitaxy using dimer arsenic, Appl. Phys. Lett. 52 (1988) 1596-1598.

[4] Tihanyi J. and SCHLOTterer H., Influence of the floating substrate potential on the characteristics of ESFI MOS transistors, Solid State Electron. 18 (1975) 309-314.

[5] Colinge J. P., Reduction of kink effect in thin film SOI MOSFET's, IEEE Electron Devices Lett. 9 (1988) 97-99.

[6] Mottet S. and Le Mouellic C., GaAs MESFET anomalies related to minority carrier injection into semi-insulating substrate, Proc. of the Third Semi-Insulating III-V Materials, Eds., D. C. Look and J. S. Blakemore (Shiva Publishing Ltd, England) 1984, pp. 406-409.

[7] Zeghbroeck B. J. V., PAtrick W., Meier H. and VetTIGER R., Parasitic bipolar effect in submicron GaAs MESFET's, IEEE Electron Devices Lett. 8 (1987) 188-190.

[8] SzE S. M., Physics of Semiconductor Devices, $2^{\text {nd }}$ Ed. (John Wiley and Sons, New-York) 1981, p. 49.

[9] Capasso F., Nahory R. E. and Pollack M. A., Temperature dependence of impact ion ionisa- tion rate in $\mathrm{GaAs}$ between 20 and $200{ }^{\circ} \mathrm{C}$, Elect. Lett. 15 (1979) 117-118.

[10] Makram-Ebeid S. and Minondo P., The role of the surface and bulk of the semi-insulating substrate in low frequency anomalies of GaAs integrated circuits, IEEE Trans. Electron Devices 32 (1985) 632-642.

[11] Viallet J. E. and Mottet S., Heterojunction under Fermi-Dirac statistics : general set of equations and steady state numerical methods, Proc. Fourth Int. Conf. on the Numerical Analysis of Semiconductor Devices and Integrated Circuits, Ed., J. J. H. Miller (Boole Press Itd, Ireland) 1985, pp. 530-535.

[12] Mottet S. and Viallet J. E., Specific simulation problem related to III-V compounds : semi-insulating materials, Proc. Fourth Int. Conf. on the Numerical Analysis of Semiconductor Devices and Integrated Circuits, Ed., J. J. H. Miller (Boole Press Ltd, Ireland) 1985, pp. 436-441.

[13] Chomette A., Deveaud B., Baudet M., Auvray P. and REGRENY A., Band discontinuities and calculations on $\mathrm{GaAs}-\mathrm{AlGaAs}$ superlattice structures, J. Appl. Phys. 59 (1986) 3835-3840.

[14] ZEBBAR N., Effet du substrat semi- isolant GaAs sur le comportement des transistors MESFET à l'arséniure de gallium, Thèse de Doctorat, USTHB Alger (1987).

[15] Kastalsky A. and Kiehl R. A., On the low temperature degradation of (AlGa)As/GaAs modulation-doped field-effect transistors, IEEE Trans. Electron Devices 33 (1986) 414-423.

[16] Godts P., Constant E., ZiMMERMAnN J. and DEPREEUW D., Investigation of influence of DX centers on HEMT operation at room temperature, Elect. Lett. 24 (1988) 937-938. 